\title{
The predatory bug Orius strigicollis shows a preference for egg-laying sites based on plant topography
}

\author{
chendi yu ${ }^{1}$, Jun Huang ${ }^{\text {Corresp., } 2}{ }^{2}$, Xiaoyun Ren ${ }^{2}$, G. Mandela Fernández-Grandon ${ }^{3}$, Xiaowei Li ${ }^{2}$, Muhammad Hafeez ${ }^{2}$, \\ Yaobin Lu ${ }^{\text {Corresp. } 2}$ \\ ${ }^{1}$ Zhejiang Academy of Agricultural Sciences, State Key Laboratory for Managing Biotic and Chemical Threats to the Quality and Safety of Agro- \\ products, Institute of Plant Protection and Microbiology, Hangzhou, Zhejiang, China \\ 2 Zhejiang Academy of Agricultural Sciences, State Key Laboratory for Managing Biotic and Chemical Threats to the Quality and Safety of Agro-products, \\ Institute of Plant Protection and Microbiology, Hangzhou, Zhejiang, China \\ 3 University of Greenwich, Natural Resources Institute, Chatham Maritime, Kent, UK \\ Corresponding Authors: Jun Huang, Yaobin Lu \\ Email address: junhuang1981@aliyun.com, luybcn@163.com
}

Background. Oviposition site selection is an important factor in determining the success of insect populations. Orius spp. are widely used in the biological control of a wide range of soft-bodied insect pests such as thrips, aphids, and mites. Orius strigicollis (Heteroptera: Anthocoridae) is the dominant Orius species in southern China; however, what factor drives its selection of an oviposition site after mating currently remains unknown.

Methods. Here, kidney bean pods (KBPs) were chosen as the oviposition substrate, and choice and nonchoice experiments were conducted to determine the preferences concerning oviposition sites on the KBPs of $O$. strigicollis. The mechanism of oviposition behavior was revealed through observation and measurement of oviposition action, the egg hatching rate, and the oviposition time.

Results. We found that $O$. strigicollis preferred the seams of the pods for oviposition, especially the seams at the tips of the KBPs. Choice and nonchoice experiments showed that females did not lay eggs when the KBP tail parts were unavailable. The rates of egg hatching on different KBP parts were not significantly different, but the time required for females to lay eggs on the tip seam was significantly lower. Decreased oviposition time is achieved on the tip seam because the insect can exploit support points found there and gain leverage for insertion of the ovipositor.

Discussion. The preferences for oviposition sites of $O$. strigicollis are significantly influenced by the topography of the KBP surface. Revealing such behavior and mechanisms will provide an important scientific basis for the mass rearing of predatory bugs. 
1 The predatory bug Orius strigicollis shows a preference for egg-laying sites

2 based on plant topography

3 Chendi $\mathrm{Yu}^{1}$, Jun Huang ${ }^{1}$, Xiaoyun Ren ${ }^{1}$, G. Mandela Fernández-Grandon ${ }^{2}$, Xiaowei Li $^{1}$, Muhammad Hafeez ${ }^{1}$ and

4 Yaobin $\mathrm{Lu}^{1}$

5

$6{ }^{1}$ State Key Laboratory for Managing Biotic and Chemical Threats to the Quality and Safety of Agro-products,

7 Institute of Plant Protection and Microbiology, Zhejiang Academy of Agricultural Sciences, Hangzhou 310021,

8 China

$9{ }^{2}$ Natural Resources Institute, University of Greenwich, Chatham Maritime, Kent, ME4 4TB, UK

10 Corresponding Author:

11 Jun Huang

12 NO. 298, Desheng Road, Hangzhou City, Zhejiang Province, 310021, China.

13 Junhuang1981@126.com

14 Yaobin LU

15 NO. 298, Desheng Road, Hangzhou City, Zhejiang Province, 310021, China.

16 luybcn@163.com

17

18

19

20

21

22

23

24

25

26

27

28

29

30

Peer] reviewing PDF | (2021:03:59021:2:0:NEW 19 Jun 2021) 


\section{Abstract}

34 Background. Oviposition site selection is an important factor in determining the success of insect populations. Orius spp. are widely used in the biological control of a wide range of soft-bodied insect pests such as thrips, aphids, and mites. Orius strigicollis (Heteroptera: Anthocoridae) is the dominant Orius species in southern

37 China; however, what factor drives its selection of an oviposition site after mating currently remains unknown.

38 Methods. Here, kidney bean pods (KBPs) were chosen as the oviposition substrate, and choice and nonchoice experiments were conducted to determine the preferences concerning oviposition sites on the KBPs of $O$. strigicollis. The mechanism of oviposition behavior was revealed through observation and measurement of

41 oviposition action, the egg hatching rate, and the oviposition time.

42 Results. We found that $O$. strigicollis preferred the seams of the pods for oviposition, especially the seams at 43 the tips of the KBPs. Choice and nonchoice experiments showed that females did not lay eggs when the KBP 44 tail parts were unavailable. The rates of egg hatching on different KBP parts were not significantly different, 45 but the time required for females to lay eggs on the tip seam was significantly lower. Decreased oviposition time is achieved on the tip seam because the insect can exploit support points found there and gain leverage for 47 insertion of the ovipositor.

48 Discussion. The preferences for oviposition sites of $O$. strigicollis are significantly influenced by the topography of the KBP surface. Revealing such behavior and mechanisms will provide an important scientific basis for the mass rearing of predatory bugs.

Keywords oviposition behavior, site selection preference, egg hatching, plant topography, Orius strigicollis

52 
64

65

66

67

68

69

70

\section{Introduction}

Insects tend to have the ability to select particular egg-laying sites to increase the survival rate of their offspring (Grostal \& Dicke, 1999; Choh \& Takabayashi, 2007; Barbosa-Andrade et al., 2019). Several factors can influence this behavior, for example, the existence of natural enemies or competitors (Rouault et al., 2007; Choh et al., 2015; Saitoh \& Choh, 2018) and site properties such as food resource availability (Bond et al., 2005), illumination intensity (Yang, 2006) and temperature (Notter-Hausmann \& Dorn, 2010). Apart from those common factors, some rare external physical factors such as the site size (Reich \& Downes, 2003), shape, or color (Markheiser et al., 2017) can also play a role in the selection of oviposition sites.

The underlying cues mentioned above are complex and are less well understood than other aspects of insect behavior (Lundgen et al., 2008). However, due to the feeding habits of phytophagous insects, plants bring importance to the life histories and agricultural value of the predators that feed on these phytophagous insects (Lundgen et al., 2008; Puysseleyr \& Hofte, 2011). Previous studies have shown that both plant species and variations in plant parts or tissues influence the oviposition behavior of predatory insects (Isenhour \& Yeargen, 1982; Coll, 1996; Lundgren \& Fergen, 2006; Pascua et al., 2019). Of the many plant morphological features, the plant physical structure is one of the important factors that is known to significantly affect this reproductive behavior, either positively (Benedict et al., 1983; Griffen \& Yeargan, 2002) or negatively (Simmons \& Gurr, 2004). The mechanisms that drive female oviposition decisions have evolved such that female insects will choose sites with the optimal plant-based resources for the survival of their offspring (Malheiro et al., 2018; Mitchell et al., 2019). However, whether other factors influence the choice of oviposition sites by predatory insects remains to be explored.

Orius spp. are widely used in biological control methods to control many pests worldwide because they exhibit a higher search efficiency for their host than other species and are fast-moving and active (Minks et al., 1989). For example, Orius strigicollis Poppius (Heteroptera: Anthocoridae) is an important native natural predator of a wide range of soft-bodied insect pests such as thrips, aphids, and mites in several agronomic systems (Cocuzza et al., 1997; Sengonca et al., 2008; Zhang et al., 2012; Bonte \& De Clercq, 2011) and feeds on lepidopteran pest eggs and young larvae (Bonte \& De Clercq, 2011; Ali et al., 2020). There are several studies about $O$. strigicollis behavior that focus on its predatory advantages and its influence on agriculture (Zhou et al., 2006; Ali et al., 2020), but the mechanisms that influence the oviposition behavior of $O$. strigicollis based on plant characteristics remain poorly understood. However, studies on another zoophytophagous heteropteran, Orius insidiosus (Say), have reported that plant species, as well as the variations within each plant, significantly influence their oviposition behavior (Coll, 1996; Lundgren \& Fergen, 2006; Pascua et al., 2019) and that they prefer to lay eggs on thinner epidermal plant surfaces, where the vesicular and cellular tissues are conducive to the survival and development of nymphs (Lundgen et al., 2008). As O. strigicollis is a natural 
97 enemy of plant pests, studying its oviposition site selection behavior will be very useful for the elaboration of 98 mass rearing protocols.

99 Kidney bean pods (hereafter KBPs) are widely used in the indoor rearing of thrips and omnivorous bugs 100 because of their freshness and convenience (Bonte \& De Clercq, 2010; Li et al., 2018). We observed that $O$. 101 strigicollis preferred KBPs for oviposition and seemed to have a preference for laying eggs on different parts 102 of the KBPs. Therefore, we used KBPs as an oviposition substrate to study the mechanism of egg-laying 103 selection preference in $O$. strigicollis. Thus, we hypothesized that there are differences in the number of eggs 104 laid by $O$. strigicollis females in the different parts of the KBPs and that the most likely mechanism driving 105 female oviposition decisions is the physical comfort of the laying position, which is directly related to the egg106 laying efficiency. Here, we attempted to answer the following questions 1) Do O. strigicollis females exhibit 107 oviposition site selection behavior, and where do females choose to lay eggs? 2) Does the presence of the bean 108 tail influence oviposition behaviors under choice and nonchoice conditions, or is the bean tip the best place for 109 O. strigicollis females to lay eggs? 3) Why do O. strigicollis females select a specific location?

\section{Materials \& Methods}

111 Insect rearing and experimental preparation

112 Orius strigicollis adults were collected from open areas and vegetable fields outside of Hangzhou

$113\left(30.43898^{\circ} \mathrm{N}, 120.41134^{\circ} \mathrm{E}\right)$, Zhejiang Province, P.R. China, and maintained in a climate-controlled room. The 114 rearing conditions were $26 \pm 2{ }^{\circ} \mathrm{C}$ and $70 \pm 10 \% \mathrm{RH}$, with a photophase of $14 \mathrm{~h}$. All growth stages of $O$.

115 strigicollis were reared in $4.3 \mathrm{~L}$ glass jars (see Supplement 1 for more details) with a circular slant (i.e., the 116 opening of the jar was on the side) capped with plastic screw-on lids. KBPs (length: $20.6 \pm 5.1 \mathrm{~cm}$ ) were used 117 in the experiments as oviposition substrates for $O$. strigicollis. From the nymph to adult stages, the predatory

118 bugs were fed western flower thrips, Frankliniella occidentalis (Pergande).

\section{Oviposition site selection preferences}

120 A pair of KBPs was laid flat on a filter paper inside the jars, and five mated $O$. strigicollis females were placed 121 into each jar and allowed to oviposit for 48 hours. Approximately $100 \mathrm{~F}$. occidentalis nymphs were placed in 122 each jar as food every day. The climatic conditions were the same as those described above. The KBPs were 123 collected 48 hours later for egg counting. The number of eggs per pod and the number of eggs in different 124 positions on the pod (face or seam) were counted under a Nikon SMZ1500 zoom stereomicroscope (Nikon, 125 Japan). The number of eggs was determined by counting the exposed opercula. The KBPs were divided into 126 three parts for this count, i.e., the tail, middle, and head (Figure 1A). A pair of KBPs was regarded as one 127 separate biological replicate, with 20 replicates in total.

\section{Influence of restricting KBP access on the oviposition site selection in Orius strigicollis}

129 The tail of the KBP was wrapped with parafilm to render the preferred oviposition site inaccessible, and then 130 nonchoice and choice testing were conducted to determine the oviposition site selection of $O$. strigicollis.

131 Nonchoice testing was conducted with the tail covered, and choice testing was also performed with the tail 
132 covered on one pod and that of another pod presented uncovered. Egg counts were conducted as described

133 above. In each test, a pair of intact KBPs inside a jar was used as a control. The nonchoice testing was

134 replicated 19 times, and the choice testing was replicated 14 times.

135 Differences in the egg number and egg hatching rate on the middle and tail of KBPs

136 To better identify the optimal oviposition site of $O$. strigicollis, we redefined the middle as the left middle

137 (Middle-L) and right middle (Middle-R), and the tail as the left tail (Tail-L) and right tail (Tail-R). The side to

138 which the kidney bean tip turns was defined as the left side, and the other side was defined as the right side

139 (Figure 1E). The tail of the KBP was then further categorized into four parts, i.e., the left neck (Neck-L), right

140 neck (Neck-R), left tip (Tip-L), and right tip (Tip-R). The narrowest part of the tip and the position where the

141 tip begins to widen (extending ca. $1.0 \mathrm{~cm}$ towards the head) were defined as the "tip", while the rest was the

142 "neck" (Figure 1F). Each pair of KBPs in each jar was considered a group, and the experiments were

143 performed again as described above. The egg numbers on each of the further-divided parts (left or right, neck

144 or tip) were counted, and the number of eggs hatched after 5 days was also recorded. If the operculum was

145 opened and no dead nymph was found around the operculum, egg hatching was considered successful. Each

146 treatment was replicated 20 times.

147 Observation of egg-laying behavior and analysis of oviposition efficiency

148 During the control experiments (i.e., a pair of intact KBPs presented for the oviposition test), the egg-laying

149 movements of 15 females on the tail and middle sections were observed, and the entire egg-laying process was

150 recorded using a micro-video recording system (HDR-SR11E, Sony, Japan). When the start of the egg-laying

151 movement was observed, an electronic timer (Deli, China) was used to determine how long females took to lay

152 one egg on the tail or middle section.

153 Statistical analysis

154 Microsoft Excel (version 16.39) was used to record the data. The analysis was conducted using Prism 8

155 (version 8.4.0) and SPSS (version 26.0). The nonparametric Wilcoxon's matched pairs test was used to

156 compare the differences in egg laid (\%) between different positions (i.e., face and seam) or different sites (i.e.,

157 tip and neck). The nonparametric Friedman's test was used to analyze the differences in egg laid (\%) between

158 different parts (i.e., head, middle, and tail) or subsections (tail-R, tail-L, middle-R, and middle-L) of the KBPs.

159 A $t$-test was used to compare the total number of eggs and the egg-laying efficiency between treatments.

\section{Results}

\section{Oviposition site selection preferences}

162 A total of $97.9 \%$ of the eggs were laid on the seam of the KBPs, and only $2.1 \%$ of the eggs were laid on the

163 face (Figure 1A; $Z=3.9, d f=19, P<0.0001$ ). Moreover, significant differences in egg numbers were

164 observed between different KBP parts. More eggs were laid on the seam of the tail and middle than on the

165 seam of the head, and the highest percentage of eggs was laid on the tail (Figure 1B; $\chi^{2}=32.7, n=20, d f=2$, 
$166 P<0.0001)$, i.e., more than half of the total eggs (50.9\%). Overall O. strigicollis females laid more eggs on the

167 seam of the KBPs, specifically on the tails.

168 Influence of restricting KBP access on the oviposition site selection in Orius strigicollis

169 A nonchoice experiment was conducted with tail coverage. In this treatment, the total number of eggs on each

170 pod was $57.7 \%$ lower than that in the control (Figure $1 \mathrm{C}$; $55.1 \pm 3.0$ vs. $130.2 \pm 7.1$ individuals, $t=11.2, d f=$

171 18, $P<0.0001)$. A choice assay was also performed with the tail covered for one pod and another pod

172 presented uncovered. In this case, the mean number of eggs per replicate was $87.6 \pm 6.73$ individuals, which

173 was also $32.7 \%$ lower than that found in the control (Figure $1 \mathrm{D} ; t=5.7, d f=13, P<0.0001$ ). The data

174 indicated that $O$. strigicollis females did not lay more eggs on other parts of the KBP when the tail parts were

175 unavailable.

176 Differences in the egg number and egg hatching rate at different oviposition sites except for the head of 177 the KBPs

178 The right tail section (Tail-R) was the most preferred by $O$. strigicollis for oviposition, followed by the left

179 middle section (Middle-L) (Figure 1E, $\chi^{2}=47.0, n=20, d f=3, P<0.0001$ ). Furthermore, it was shown that

180 the tip section was the most preferred by $O$. strigicollis for laying eggs (Figure 1F; $Z=3.9, d f=19, P<$

181 0.0001). The egg hatching rates on different sections were not significantly different, and all were higher than

$18280 \%$ (Figure $2 \mathrm{~A}, \chi^{2}=3.4, n=20, d f=4, P=0.489$ ). Therefore, we indicate that factors such as the hatching or 183 survival of eggs may not influence the selection of oviposition sites.

184 Observation of egg-laying behavior and efficiency analysis

185 The average time that each female spent laying one egg on the tip was $28.7 \%$ shorter than the time spent to lay 186 an egg on the middle section (Figure $2 \mathrm{~B}, t=6.0, d f=14, P<0.0001$ ). We observed that laying eggs on the 187 right tip seam was more efficient than laying eggs in another section (Figure 2C/D), which indicates that the 188 more uneven the plant surface is, the more conducive it is to $O$. strigicollis oviposition.

189 Discussion

190 Postmating behavior such as oviposition site selection is observed in many insect species and is important for 191 the reproduction of these species (Thompson, 1988). For example, Gryllus texensis Cade and Otte and some 192 myrmecophilous butterfly species choose a suitable oviposition site for the survival of their offspring

193 (Stahlschmidt \& Adamo, 2013). In this study, we found that $O$. strigicollis females selected the seam of the 194 KBPs rather than the face for egg laying. Such a preference difference for a different site on the same type of 195 tissue or unit is common in oviposition site selection. For example, the lepidopteran multivoltine leafminers

196 Phyllocnistis sp. prefer to lay eggs on only the lower-surface epidermal layer of the primary shoots, switching 197 to lamma shoots when they appear later in the season (Ayabe et al., 2017). The longhorn beetle, Glenea cantor 198 (F.), preferentially selects the upper section of kapok trees first for oviposition according to the bark moisture 199 content from the top to the bottom of the trees (Lu et al., 2011). For insects with endophytic oviposition, the 200 effects of characteristics of the plant tissues on their oviposition preference are more obvious (Lundgen et al., 
201 2008). For example, Pascua et al. (2019) found that the strawberry calyx and flower petiole received more

202 eggs than the other structures, and the thickness of the external tissues did not affect the oviposition of

203 O. insidiosus. Likewise, Isenhour \& Yeargan (1982) recorded a greater number of O. insidiosus eggs in the

204 petiole of soybean flowers than in the other structures of the plant. Additionally, in our study, we found more

205 than half of $O$. strigicollis eggs in the tip part, which was the primary oviposition site compared with other

206 parts of the KBP. Further experiments were conducted to elucidate the hierarchy of preference of egg-laying

207 females and identify the factors that influence it.

208 The results of the choice experiments suggest that first, when one of the preferred parts was unavailable, the

209 total number of eggs laid decreased. Second, when none of the preferred parts were available the number of

210 total eggs laid decreased rapidly, and although the left middle seam remained available for oviposition this site

211 did not replace the preferred site. A previous study on mosquitoes suggested that the decreased oviposition rate

212 observed on highly enriched leaves may be due to a pungent odor that is caused by the extreme anoxic

213 environment and repels gravid female mosquitoes (Hoekman et al., 2007). Similar behavior was also observed

214 in peach twig borers, Anarsia lineatella Zeller, of which female adults can determine whether peach fruits are

215 fresh and viable for oviposition so that their larvae can have enough time to develop into adults before the

216 peach fruits decompose (Sidney et al., 2008). In addition to odor and freshness, the sweetness and hardness of

217 substrates also affected the egg-laying site selection of insects. Wu et al. (2019) showed that activation of

218 sweet neurons by sucrose can promote Drosophila females to become indifferent between two substrates of

219 different hardness levels during egg laying. In this study, we assumed that the seam of KBP might be a good

220 place since the eggs would remain more hidden, allowing it to provide a kind of refuge and that in the extreme

221 (tip), the tissues may be softer than in the middle. This could also be related to oviposition efficiency.

222 However, regardless of the reason for the egg-laying site selection, insects choose to lay eggs on well-

223 nourished hosts or tissue to ensure the healthy development and survival of their offspring (Jeong et al., 2016;

224 Malheiro et al., 2018; Mitchell et al., 2019). Here, we found that O. strigicollis laid the most eggs at the tip of

225 the KBPs, which indicated this location as their preferred oviposition site. The egg hatching rate is an

226 important biological index used to measure host fitness or the suitability of oviposition substrates (Murai et al.,

227 2001; Bonte \& Clercq, 2010; Krug \& Sosa, 2019), and it is also the most intuitive criterion to judge (Castane

$228 \&$ Zalom, 1994). Therefore, we further analyzed the hatching rates of eggs laid on different parts of the KBPs

229 (tail vs. middle). The data showed that the hatching rates on these four sections (middle $\mathrm{L}$ and $\mathrm{R}$, tail $\mathrm{L}$ and $\mathrm{R}$ )

230 were not significantly different. We suggest that the factors that influence the selection of oviposition sites may

231 not be those that restrict the hatching or survival of eggs. Additionally, we found that the eggs were embedded

232 in the KBP tissue, and the lid of the egg was opened when it hatched. Embedding eggs may simply protect the

233 eggs from predation or parasitism and from abiotic factors, in addition to stabilizing the eggs or keeping them

234 in a moist environment (Shapiro \& Ferkovich, 2006). According to the optimal oviposition theory, plant tissue

235 acceptability for oviposition may also be affected by the subsequent development or survival of Orius nymphs

236 (Jaenike, 1978; Lundgren \& Fergen, 2006). It is known that in many Orius species newly emerged nymphs

Peer] reviewing PDF | (2021:03:59021:2:0:NEW 19 Jun 2021) 
237 use plant tissues to gain energy and begin their dispersal. Perhaps in the tip part of the KBPs, the tissues are

238 softer or have some characteristics that make it easier for the small nymphs to feed more easily, but this

239 hypothesis is worth further systematic study.

240 Based on our observation of the entire egg-laying process of the females and our measurements of the time

241 required for the females to lay eggs, we suggest that females select the tip of the KBPs as their first oviposition

242 site to achieve higher egg-laying efficiency, and reducing their time spent ovipositing also reduces their risk of

243 predation and allows more time for foraging and perching (Martens, 2001; Philippe et al., 2015). Furthermore,

244 we suggest that the increased egg-laying efficiency is due to the 'ergonomics' of this egg-laying position. The

245 females must use force to insert their eggs into the KBP. To achieve this, they need anchor points for both their

246 propodeum and metapodium to push against to gain the required power. Comparing the seam at the tip and in

247 the middle section of the KBPs, the females were able to clasp the tip of the KBPs using their propodeum. This

248 allowed oviposition in KBPs with much greater ease (see Supplement 2 for more details). In contrast, because

249 the side of the KBP is nearly flat, the females are required to use more strength and expend more energy to

250 insert their eggs there. There is a similar explanation for the low egg distribution on the seam on the other

251 side - compared with the preferred seam, the other seam is relatively shallow, and more energy might be

252 required for the females to lay their eggs inside it. Similar observations and speculations were also mentioned

253 briefly by Shapiro \& Ferkovich (2006), who speculated that female adults of $O$. insidiosus may need to take

254 advantage of the internal angles or surface irregularities to gain leverage for the ovipositor.

\section{Conclusions}

256 In this study, according to different positions and parts, the KBPs (oviposition substrates) were divided into

257 different sites, e.g., face, seam, tail (including the neck and tip), middle, and head. We found that the physical

258 features of each site were ultimately reflected in the corresponding egg-laying efficiency. The results suggest

259 that the preference for oviposition sites of $O$. strigicollis is significantly influenced by the topography of the

260 KBP surface, and the more 'comfortable' the females are, the higher their egg-laying efficiency. The

261 behavioral mechanism of the preference of $O$. strigicollis females for oviposition sites on the KBPs was found

262 and identified, which is conducive to our later development of artificial media to attract $O$. strigicollis to lay

263 eggs. The results also provide the necessary knowledge to advance the massive production of $O$. strigicollis for

264 their release within the framework of an augmentative biological control strategy.

265

\section{Acknowledgments}

267 This study was supported by the Project National Key Research and Development (2017YFD0201000), and

268 the National Natural Science Foundation of China (31772234, 31801801, 31672031).

269

\section{References}


271

272

273

274

275

276

277

278

279

280

281

282

283

284

285

286

287

288

289

290

291

292

293

294

295

296

297

298

299

300

301

302

303

304

305

306

Ali S, Zhu Q, Jaleel W, Rehman SU, Rasheed MA, Khan MM, Islam Y, Hafeez M, Zhou X. 2020. Determination of fitness traits of Orius strigicollis Poppius (Hemiptera: Anthocoridae) on Pectinophora gossypiella (Lepidoptera: Gelechiidae) using two-sex life table analysis. PeerJ 8: e9594 DOI: $10.7717 /$ peerj.9594

Ayabe Y, Minoura T, Hijii N. 2017. Oviposition site selection by a lepidopteran leafminer in response to heterogeneity of leaf surface conditions: structural traits and microclimates: Leafminer oviposition site selection. Ecol Entomol 42: 294-305 DOI 10.1111/een.12387

Barbosa-Andrade CL, Andrade DJ, Matos STS, Nogueira L, Cividanes FJ. 2019. Selection of oviposition sites by ground beetles Abaris basistriata and Selenophorus seriatoporus for mass rearing purposes. BioControl 64: 315-321 DOI 10.1007/s10526-019-09930-8

Benedict JH, Leigh TF, Hyer AH. 1983. Lygus hesperus (Heteroptera: Miridae) oviposition behavior, growth, and survival in relation to cotton trichome density. Environ Entomol 12: 331-335 DOI 10.1093/ee/12.2.331

Bond JG, Arredondo-Jimenez JI, Rodriguez MH, Quiroz-Martinez H, Williams T. 2005. Oviposition habitat selection for a predator refuge and food source in a mosquito. Ecol Entomol 30: 255-263 DOI 10.1111/j.0307-6946.2005.00704.x

Bonte M, De Clercq P. 2010. Impact of artificial rearing systems on the developmental and reproductive fitness of the predatory bug, Orius laevigatus. J Insect Sci 10: 104 DOI 10.1673/031.010.10401

Bonte M, De Clercq P. 2011. Influence of predator density, diet and living substrate on developmental fitness of Orius laevigatus. J Appl Entomol 135: 343-350 DOI 10.1111/j.1439-0418.2010.01554.x

Castane C, Zalom FG. 1994. Artificial oviposition substrate for rearing Orius insidiosus (Hemiptera, Anthocoridae). Biol Control 4: 88-91 DOI 10.1006/bcon.1994.1015

Choh Y, Sabelis MW, Janssen A. 2015. Distribution and oviposition site selection by predatory mites in the presence of intraguild predators. Exp Appl Acarol 67: 477-491 DOI 10.1007/s10493-015-9970-8

Choh Y, Takabayashi J. 2007. Predator avoidance in phytophagous mites: response to present danger depends on alternative host quality. Oecologia 151: 262-267 DOI 10.1007/s00442-006-0590-1

Cocuzza GE, Clercq PD, De Veire MV, Cock AD, Degheele D, Vacante V. 1997. Reproduction of Orius laevigatus and Orius albidipennis on pollen and Ephestia kuehniella eggs. Entomol Exp Appl 1: 101104 DOI 10.1046/j.1570-7458.1997.00118.x

Coll M. 1996. Feeding and ovipositing on plants by an omnivorous insect predator. Oecologia 105: 214220 DOI 10.1007/BF00328549

Griffen ML, Yeargan KV. 2002. Factors potentially affecting oviposition site selection by the lady beetle Coleomegilla maculate (Coleoptera: Coccinellidae). Environ Entomol 31: 112-119 DOI 10.1603/0046-225X-31.1.112

Grostal P, Dicke M. 1999. Direct and indirect cues of predation risk influence behavior and reproduction of prey: a case for acarine interactions. Behav Ecol 10: 422-427 DOI 10.1603/0046-225X-31.1.112

Peer] reviewing PDF | (2021:03:59021:2:0:NEW 19 Jun 2021) 
307

308

309

310

311

312

313

314

315

316

317

318

319

320

321

322

323

324

325

326

327

328

329

330

331

332

333

334

335

336

337

338

339

340

341

Hoekman D, Terhorst C, Bauer A, Braun S, Gignac P, Hopkins R, Joshi S, Laskis K, Sanscrainte N, Travis J, Miller TE. 2007. Oviposition decreased in response to enriched water: a field study of the pitcher-plant mosquito, Wyeomyia smithii. Ecol Entomol 32: 92-96 DOI 10.1111/j.13652311.2006.00840.x

Isenhour DJ, Yeargan KV. 1982. Oviposition sites of Orius insidiosus (Say) and Nabis spp. in soybean (Anthocoridae and Nabidae). J Kansas Entomol Soc 55: 65-72 DOI 10.1016/0022-474X(82)90029-7

Jaenike J. 1978. On optimal oviposition behavior in phytophagous insects. Theor Popul Biol 14: 350-356 DOI 10.1016/0040-5809(78)90012-6

Jeong YT, Oh SM, Shim J, Seo JT, Kwon JY, Moon SJ. 2016. Mechanosensory neurons control sweet sensing in Drosophila. Nat Commun 7: 12872 DOI 10.1038/ncomms 12872

Krug P, Sosa AJ. 2019. Mother knows best: plant polyploidy affects feeding and oviposition preference of the alligator weed biological control agent, Agasicles hygrophila. BioControl 64: 623-632 DOI 10.1007/s10526-019-09959-9

Li X, Geng S, Zhang Z, Zhang J, Li W, Huang J, Lin W, Bei Y, Lu Y. 2018. Species-specific aggregation pheromones contribute to coexistence in two closely related thrips species. B Entomol Res 109: 119-126 DOI 10.1017/S0007485318000366

Lu W, Wang Q, Tian MY, Xu J, Qin AZ, He L, Jia B, Cai JJ. 2011. Host selection and colonization strategies with evidence for a female produced oviposition attractant in a longhorn beetle. Environ Entomol 40: 1487-1493 DOI 10.1603/EN10280

Lundgren JG, Fergen JK. 2006. The oviposition behavior of the predator Orius insidiosus: acceptability and preference for different plants. BioControl 51: 217-227 DOI 10.1007/s10526-005-0609-2

Lundgen JG, Fergen JK, Riedell WE. 2008. The influence of plant anatomy on oviposition and reproductive success of the omnivorous bug Orius insidiosus. Anim Behav 75: 1495-1502 DOI 10.1016/j.anbehav.2007.09.029

Markheiser A, Rid M, Biancu S, Gross J, Hoffmann C. 2017. Physical factors influencing the oviposition behaviour of European grapevine moths Lobesia botrana and Eupoecilia ambiguella. $J$ Appl Entomol 142: 201-210 DOI 10.1111/jen.12423

Martens A. 2001. Initial preference of oviposition sites: discrimination between living and dead plant material in Sympecma fusca and Coenagrion caerulescens (Odonata: Lestidae, Coenagrionidae). Eur J Entomol 98: 121-123 DOI 10.14411/eje.2001.021

Malheiro R, Susana C, Pinheiro L, Baptista P, Pereira J. 2018. Olive cultivar and maturation process on the oviposition preference of Bactrocera oleae (Rossi) (Diptera: Tephritidae). B Entomol Res 109: 1-11 DOI 10.1017/S0007485318000135

Minks AK, Harrewijg P, Helle W. 1989. Aphids: Their biology, natural enemies and control. Q Rev Biol 64(2): 208-209 DOI 10.1016/0378-4290(89)90065-8

Peer) reviewing PDF | (2021:03:59021:2:0:NEW 19 Jun 2021) 
342

343

344

345

346

347

348

349

350

351

352

353

354

355

356

357

358

359

360

361

362

363

364

365

366

367

368

369

370

371

372

373

374

375

376

Mitchell TS, Shephard AM, Kalinowski CR, Kobiela ME, Snell-Rood EC. 2019. Butterflies do not alter oviposition or larval foraging in response to anthropogenic increases in sodium. Anim Behav 154: 121129 DOI 10.1016/j.anbehav.2019.06.015

Murai T, Naraim Y, Sugiura N. 2001. Utilization of germinated broad bean seeds as an oviposition substrate in mass rearing of the predatory bug, Orius sauteri (Poppius) (Heteroptera: Anthocoridae). Appl Entomol Zool 36: 489-494 DOI 10.1303/aez.2001.489

Notter-Hausmann C, Dorn S. 2010. Relationship between behavior and physiology in an invasive pest species: Oviposition site selection and temperature-dependent development of the oriental fruit moth (Lepidoptera: Tortricidae). Environ Entomol 39: 561-569 DOI 10.1603/EN09231

Pascua M, Rocca M, De Clercq P, Greco N. 2019. Host plant use for oviposition by the insidious flower bug (Hemiptera: Anthocoridae). J Econ Entomol 112(1): 219-225 DOI 10.1093/jee/toy310

Philippe L, Besnard A, Natalia M. 2015. Initial preference for plant species and state during oviposition site selection by an odonate. Entomol Sci 18: 377-382 DOI 10.1111/ens.12130

Puysseleyr VD, Hofte M. 2011. Ovipositing Orius laevigatus increase tomato resistance against Frankliniella occidentalis feeding by inducing the wound response. Arthropod-Plant Inte 5: 71-80 DOI 10.1007/s11829-010-9117-0

Reich P, Downes B. 2003. Experimental evidence for physical cues involved in oviposition site selection of lotic hydrobiosid caddis flies. Oecologia 136: 465-475 DOI 10.1007/s00442-003-1284-6

Rouault G, Battisti A, Roques A. 2007. Oviposition sites of the cypress seed bug Orsillus maculatus and response of the egg parasitoid Telenomus gr. Floridanus. BioControl 52: 9 DOI 10.1007/s10526-006$9002-z$

Saitoh F, Choh Y. 2018. Do intraguild prey protect their eggs from intraguild predators that share their oviposition site? Anim Behav 140: 49-55 DOI 10.1016/j.anbehav.2018.04.005

Sengonca C, Ahmadi K, Blaeser P. 2008. Biological characteristics of Orius similis Zheng (Heteroptera, Anthocoridae) by feeding on different aphid species as prey. J Plant Dis Protect 115: 32-38 DOI 10.1098/rspb.2006.3676

Shapiro JP, Ferkovich SM. 2006. Oviposition and isolation of viable eggs from Orius insidiosus in a Parafilm and water substrate: Comparison with green beans and use in enzyme-linked immunosorbent assay. Ann Entomol Soc Am 99: 586-591 DOI 10.1038/sj.ijo.0802417

Sidney M, Brown K, Judd GJR, Gries G. 2008. Stimuli affecting selection of oviposition sites by female peach twig borer, Anarsia lineatella Zeller (Lepidoptera: Gelechiidae. J Appl Entomol 132: 538-544 DOI 10.1111/j.1439-0418.2008.01292.x

Simmons AT, Gurr GM. 2004. Trichome-based host plant resistance of Lycopersicon species and the biocontrol agent Mallada signata: are they compatible? Entomol Exp Appl 113: 95-101 DOI 10.1111/j.0013-8703.2004.00210.x

Peer] reviewing PDF | (2021:03:59021:2:0:NEW 19 Jun 2021) 
377

378

379

380

381

382

383

384

385

386

387

388

389

390

391

392

Stahlschmidt ZR, Adamo SA. 2013. Warm and cozy: temperature and predation risk interactively affect oviposition site selection. Anim Biol 86: 553-558 DOI 10.1016/j.anbehav.2013.06.009

Thompson JN. 1988. Evolutionary ecology of the relationship between oviposition preference and performance of offspring in phytophagous insects. Entomol Exp Appl 47: 3-14 DOI 10.1111/j.15707458.1988.tb02275.x

Wu SF, Ja YL, Zhang YJ, Yang CH. 2019. Sweet neurons inhibit texture discrimination by signaling TMC-expressing mechanosensitive neurons in Drosophila. eLife 8: e46165 DOI 10.7554/eLife.46165

Yang LH. 2006. Periodical cicadas use light for oviposition site selection. Proc R Soc B 273: 2993-3000 DOI 10.1098/rspb.2006.3676

Zhang S, Zhu F, Zheng X, Lei C, Zhou X. 2012. Survival and developmental characteristics of the predatory bug Orius similis (Hemiptera: Anthocoridae) fed on Tetranychus cinnabarinus (Acari: Tetranychidae) at three constant temperatures. Eur $J$ Entomol 109: 503-508 DOI 10.14411/eje.2012.063

Zhou XM, Zhu F, Li H, Lei CL. 2006. Effect of temperature on development of Orius similis Zheng (Hemiptera: Anthocoridae) and on its predation activity against Aphis gossypii Glover (Hemiptera: Aphididae). Pan Pacific Entomologist 82: 97-102. 


\section{Figure 1}

The preference of oviposition sites on kidney bean pods (KBPs) in Orius strigicollis.

A) The percentage of eggs laid on the seam and face of the KBPs. Each bar represents the mean + SEM ( $N$ $=20)$. $* *$ indicates a significant difference $(P<0.0001$, the nonparametric Wilcoxon's matched pairs test).

$B)$ The percentage of eggs laid in the head, middle and tail parts from the seam of KBPs. Different letters indicate significant differences ( $P<0.05$, the nonparametric Friedman's test). C) Comparing of the mean number of eggs (+ SEM) laid on treatment (tail covered or restricting access) and the control (uncovered) by nonchoices assay $\left(N_{\text {control }}=20, N_{\text {treatment }}=19\right) .{ }^{* *}$ indicates significant differences $(P<0.0001$, Student's $t$ test). D) Comparing of the mean number of eggs (+ SEM) laid on treatment (tail covered or restricting access) and the control (uncovered) by choices assay $\left(N_{\text {control }}=20, N_{\text {treatment }}=14\right)$. ${ }^{* *}$ indicates significant differences $(P<0.0001$, Student's $t$-test). E) Comparison of the percentage of eggs laid on the right and left sides of KBP tail and middle sections. Each bar represents the mean + SEM $(N=20)$; different letters indicate significant differences $(P<0.05$, the nonparametric Friedman's test). F) Comparison of the percentage of eggs laid on the tip and neck sections. Each bar represents the mean + SEM $(N=20)$; ** indicates significant differences $(P<0.0001$, the nonparametric Wilcoxon's matched pairs test). 
A

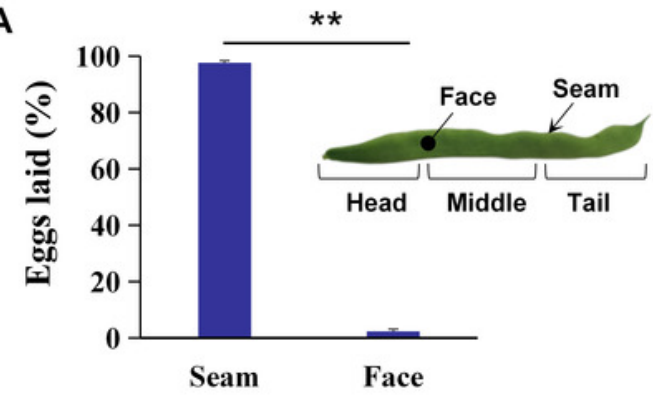

\section{C-nonchoice}

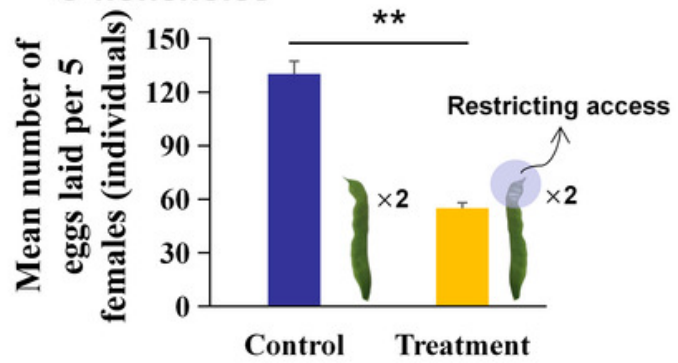

E

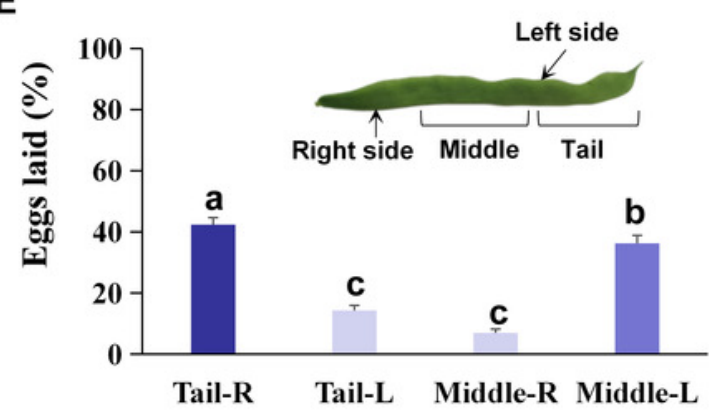

B

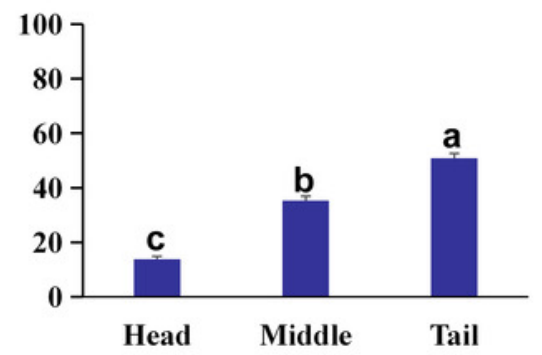

\section{D-choice}
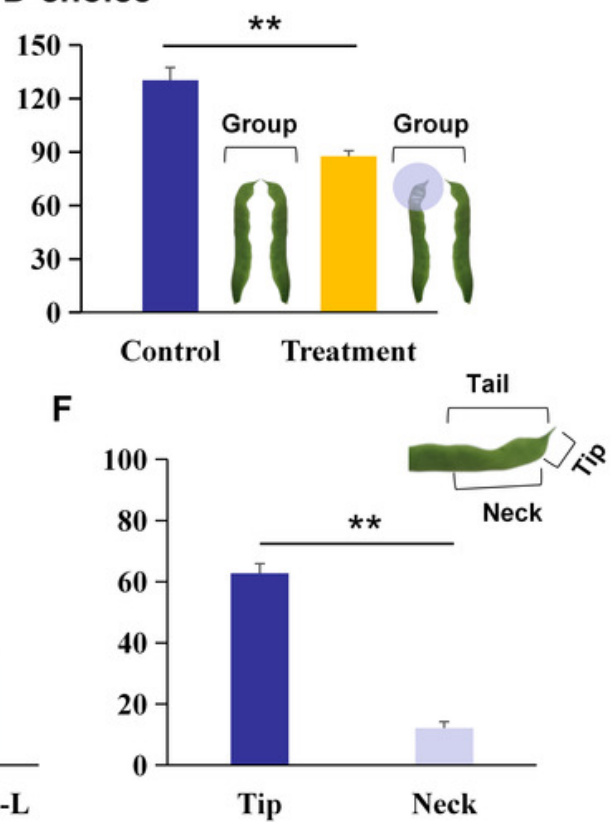


\section{Figure 2}

Behavioral mechanisms of oviposition site selection on kidney bean pods (KBPs) in Orius strigicollis.

A) Percentage of eggs hatched of eggs laid on five parts, i.e., tip, neck, right middle, left middle, and left tail. Same letters indicate no significant differences $(P>0.05$, the nonparametric Friedman's test). Each bar represents the mean + SEM $(N=20)$. B) The time spent (seconds) for females to lay one egg on the right tip and left middle sections. Each bar represents the mean + SEM $(N=15) . * *$ indicates significant differences $(P<0.0001$, Student's $t$-test). $C / D)$ Photograph of female adult ready to lay eggs on the seam of the middle and tip sections.

A

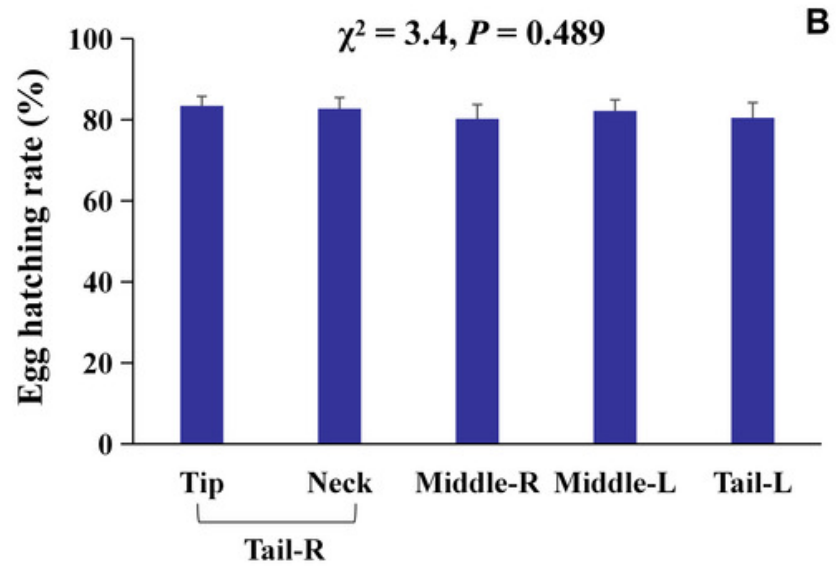

B

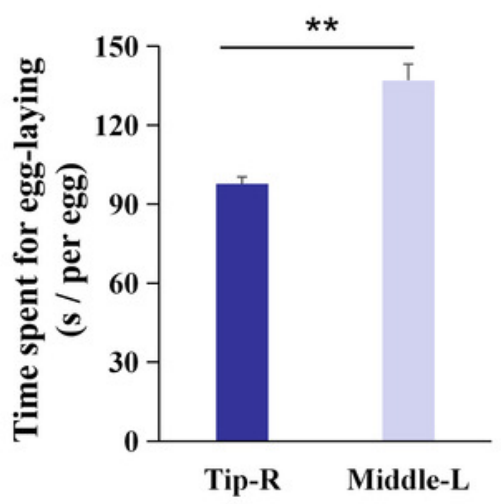

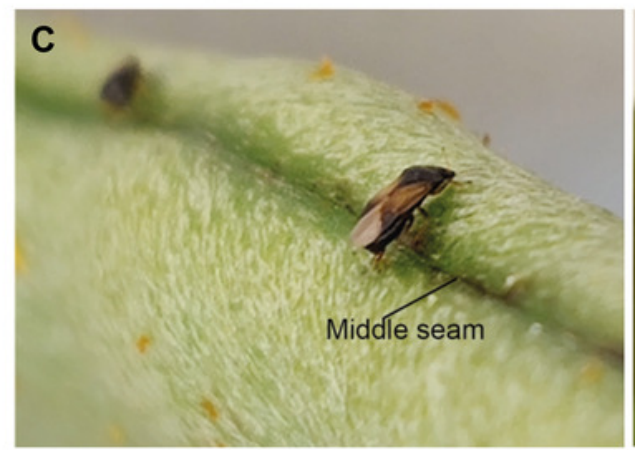

D

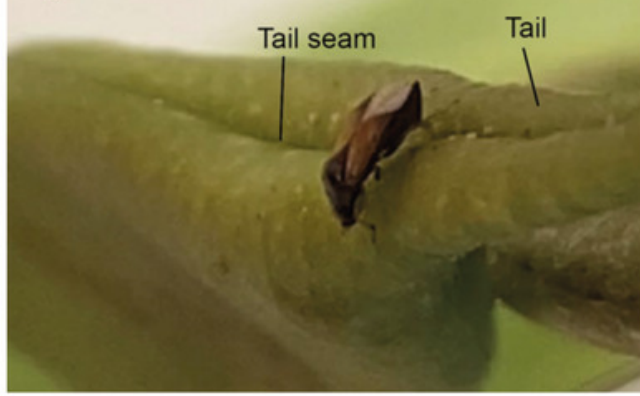

